\title{
Fatores Contingenciais e Indicadores de Desempenho: Um Estudo no Polo Calçadista de Campina Grande - PB
}

\author{
Josélia Fernandes do Nacimento \\ Mestrado em andamento em Ciências Contábeis pela Universidade Federal da Paraíba \\ - UFPB \\ Campus I - Lot. Cidade Universitaria/PB. CEP: 58.051-900 \\ E-mail: joselyafernandes@hotmail.com \\ Aldo Leonardo Cunha Callado
Doutorado em Agronegócios pela Universidade Federal do Rio Grande do Sul -
UFRGS
Professor da Universidade Federal da Paraíba - UFPB
Campus I - Lot. Cidade Universitaria/PB. CEP: 58.051-900
E-mail: aldocallado@yahoo.com.br
}

\section{RESUMO}

Este estudo tem como objetivo investigar as relações entre fatores contingenciais de empresas do polo calçadista de Campina Grande-PB e o nível de importância atribuída ao uso de indicadores de desempenho. Para a operacionalização da pesquisa, foi utilizado um questionário estruturado composto por indicadores de desempenho classificados nas dimensões do Balanced Scorecard (BSC) e fatores contingenciais relacionados com as características dos gestores, das empresas e dos critérios de mensuração de desempenho. Foram contatadas as empresas cadastradas no Sindicato da Indústria de Calçados do Estado da Paraíba, que fazem parte do polo de Campina Grande, das quais 13 empresas aceitaram participar da pesquisa. Para analisar as relações entre as variáveis, foi utilizado o coeficiente de correlação de Spearman. As evidências encontradas permitem observar que os fatores relacionados às características das empresas apresentam associações em maior proporção que os demais fatores investigados no nível de importância atribuída aos indicadores de desempenho, principalmente nas dimensões de clientes e de processos internos. As características dos gestores apresentaram poucas correlações significativas, destacando-se na dimensão financeira. E com relação às características dos critérios de mensuração, as correlações estatisticamente significativas foram encontradas em maior proporção na perspectiva de processos internos. Pode-se inferir ainda que os fatores investigados apresentam possíveis relações em maior proporção no nível de importância dada aos indicadores não financeiros.

Palavras-chave: Fatores Contingenciais. Indicadores de Desempenho. Setor Calçadista.

Contingency Factors and Performance Indicators: A Study in the Campina Grande Footwear Pole - PB 
Fatores Contingenciais e Indicadores de Desempenho: Um Estudo no Polo Calçadista de Campina

Grande - PB

Josélia Fernandes do Nacimento, Aldo Leonardo Cunha Callado

\section{ABSTRACT}

This study aims to investigate the relationship between contingency factors of companies of the Campina Grande-PB footwear polo and the level of importance attributed to the use of performance indicators. For the operationalization of the research, we used a structured questionnaire composed of performance indicators classified in the dimensions of the Balanced Scorecard (BSC) and contingency factors related to the characteristics of managers, companies and performance measurement criteria. We contacted the companies registered in the Footwear Industry Union of the State of Paraíba and that are part of the Campina Grande hub, of which 13 companies agreed to participate in the research. To analyze the relationships between the variables, the Spearman correlation coefficient was used. The evidence shows that the factors related to the characteristics of companies present associations in greater proportion than the other factors investigated, in the level of importance attributed to performance indicators, especially in the dimensions of customers and internal processes. The characteristics of managers showed few significant correlations, standing out in the financial dimension. And regarding the characteristics of the measurement criteria, statistically significant correlations were found in greater proportion from the perspective of internal processes. It can also be inferred that the factors investigated present possible relationships in greater proportion in the level of importance given to non-financial indicators.

Keywords: Contingency Factors. Performance indicators. Footwear Industry.

\section{INTRODUÇÃO}

Diante das mudanças que vêm ocorrendo no ambiente dos negócios, o qual se apresenta cada vez mais mutável e imprevisível, surge a necessidade de as organizações adotarem uma postura estratégica a fim de se manterem competitivas. Assim, as empresas fazem uso de instrumentos gerenciais, métodos e ferramentas para melhorarem seus processos de gestão e, consequentemente, o seu desempenho.

Para Zilber e Fischmann (2002), os indicadores de desempenho configuram um dos instrumentos capazes de auxiliar na determinação das estratégias empresariais, monitorando o cumprimento de objetivos e metas estabelecidos, permitido realizarem verificação e avaliação das decisões tomadas a fim de corrigir e readequar o processo vigente de gestão para que a organização alcance o melhor desempenho. 
Fatores Contingenciais e Indicadores de Desempenho: Um Estudo no Polo Calçadista de Campina

Cabe destacar que nesse cenário ainda se exige a revisão de vários preceitos, dentre eles o contexto organizacional, que é determinado por diversos fatores, assim como a forma de se avaliar o desempenho, visto que a implementação de ferramentas como os indicadores de desempenho para serem efetivas e obterem desempenho satisfatório, precisam alinhar suas estruturas às características do ambiente de atuação (Donaldson, 2001).

Hyvönen (2007) destaca que não existe uma estrutura universal e ideal para todos os tipos de organizações. Nesse sentindo, segundo Harland (1997), à medida que as especificidades das empresas são consideradas, o critério adotado para a escolha de indicadores de desempenho a serem utilizados assume uma significativa importância. Diante desse contexto, a teoria da contingência tem fornecido um paradigma coerente para a análise da estrutura das organizações, pois investiga os determinantes dos comportamentos organizacionais.

Nessa perspectiva, Hoque (2004) afirma que, por possuírem diferentes ambientes operacionais, as empresas apresentarão iniciativas estratégicas distintas; portanto, podem exigir diferentes sistemas de informação gerencial para melhorar o desempenho organizacional. Segundo Otley (2016), a adoção de práticas gerenciais pode sofrer influência de diversos fatores contingenciais que foram classificados ao longo dos anos, como fatores internos e externos à organização. Dentre esses fatores, estão estrutura organizacional, ambiente, tamanho, estratégia, tecnologia, cultura, entre outros. Com isso, as medidas de avaliação de desempenho também devem refletir as peculiaridades de cada ambiente.

Diante desse contexto, para se adaptarem às modificações enfrentadas em um ambiente competitivo, as empresas dos mais diversos setores econômicos devem dispor de indicadores de desempenho que permitam verificação da eficiência e/ou eficácia das decisões tomadas pela empresa. Assim, acredita-se que a utilização de indicadores de desempenho provavelmente também se faz presente no contexto de muitas empresas do setor calçadista, visto que a fabricação de calçados é uma atividade muito presente no desenvolvimento econômico brasileiro, com grande 
Fatores Contingenciais e Indicadores de Desempenho: Um Estudo no Polo Calçadista de Campina Grande - PB Josélia Fernandes do Nacimento, Aldo Leonardo Cunha Callado

representatividade na geração de emprego e de renda, apresentando ainda impacto no mercado de exportações.

De acordo com a Associação Brasileira das Indústrias de Calçados - Abicalçados (2017), o Brasil está entre os dez maiores produtores e consumidores de calçados no mundo. O setor é fortemente caracterizado pela concentração regional e, ao identificar a produção das regiões brasileiras, em 2017, o Nordeste destaca-se como o grande produtor de calçados no Brasil, com $58,2 \%$ da produção nacional. A Paraíba é o segundo maior polo produtor de calçados do Brasil, sendo que $92,1 \%$ da produção do estado estão concentrados no polo de Campina Grande.

O setor se destaca devido a sua significativa participação na economia. Além desse aspecto, o mesmo se encontra inserido em um mercado caracterizado pela grande concorrência nacional e internacional, o que tende a pressionar pela adoção de ferramentas associadas à gestão dos negócios. Dessa forma, o objetivo deste estudo consistiu em investigar as relações entre fatores contingenciais de empresas do polo calçadista de Campina Grande-PB e o nível de importância atribuída pelos gestores ao uso de indicadores de desempenho.

\section{REFERENCIAL TEÓRICO}

\subsection{Fatores contingenciais}

Sob a lente da teoria da contingência, não existe uma estrutura única e apropriada que se aplique a todas as organizações, pois dependerão de circunstâncias específicas do ambiente em que cada organização está inserida (Otley, 2016). Desse modo, a teoria da contingência enfatiza que a organização é um sistema aberto, e as condições do ambiente transformam a estrutura das organizações. De acordo com Donaldson (2001), as contingências podem ser entendidas como qualquer variável que modera $\mathrm{o}$ efeito de uma característica organizacional. Consequentemente, a abordagem contingencial busca captar a influência dessas variáveis nas empresas, 
Fatores Contingenciais e Indicadores de Desempenho: Um Estudo no Polo Calçadista de Campina Grande - PB Josélia Fernandes do Nacimento, Aldo Leonardo Cunha Callado

assim como o impacto no funcionamento das organizações e no desempenho das mesmas.

Espejo (2008) enfatiza que os processos organizacionais podem sofrer influência por fatores contingenciais tanto do ambiente organizacional interno quanto do ambiente externo. Beuren e Macahon (2011) reforçam que os sistemas de uma organização devem ser desenvolvidos de acordo com a adequação dos fatores externos e das características internas das empresas. Com isso, as variáveis do ambiente guiarão as decisões a serem tomadas e, conforme a organização melhor se adequa às variáveis, os objetivos podem ser atingidos de forma mais eficaz e, consequentemente, obter melhores resultados.

Silva, Scarpin Rocha e Di Domenico (2014) corroboram, afirmando que as empresas devem buscar um constante equilíbrio entre o atendimento das necessidades internas e a adaptação às circunstâncias ambientais externas, a fim de maximizar o seu desempenho. Portanto, a premissa que a teoria da contingência aborda é que quanto maior a adequação entre ambiente externo e variáveis internas, melhor será o desempenho da organização.

A literatura relacionada com o tema apresenta diversos fatores contingenciais que afetam as organizações. Otley (2016) realizou uma revisão literária sobre a teoria da contingência no período de 1980 a 2014 e agrupou as principais variáveis contingenciais pesquisadas em variáveis externas e internas. No contexto externo, destacou-se a tecnologia, competição de mercado ou hostilidade, incerteza ambiental e cultura nacional. No ambiente interno, as variáveis mais estudadas foram tamanho da organização, estrutura, estratégia, sistemas de informação, variáveis psicológicas, a participação no controle de sistemas, posição no mercado, estágio do ciclo de vida do produto e mudança de sistemas. Estas variáveis foram comumente relacionadas com as variáveis de desempenho, medidas de desempenho, comportamento orçamentário, sistema de controle, eficácia, satisfação no trabalho, mudança nas práticas e inovação de produtos. 
Fatores Contingenciais e Indicadores de Desempenho: Um Estudo no Polo Calçadista de Campina Grande - PB Josélia Fernandes do Nacimento, Aldo Leonardo Cunha Callado

Otlye (2016) ressalta que, mesmo que, nas últimas décadas, os estudos tenham apresentado diversos fatores contingenciais inseridos nos mais diferentes contextos organizacionais, atualmente a abordagem contingencial deve ser considerada em um contexto muito mais dinâmico, sendo necessário usar mais modelos baseados em processos que examinem os mecanismos de mudança e a implementação de formas modificadas de gerenciamento e controle das organizações.

Denota-se que a teoria da contingência busca encontrar a relação entre o contexto organizacional e as contingências específicas. Esta relação é capaz de auxiliar o gestor a atingir os objetivos da empresa e obter melhores resultados. No entanto, como não existe uma estrutura geral aplicável em todas as organizações e em todas as circunstâncias, pois cada estrutura organizacional é única e resultante de um conjunto de contingências, as organizações devem permanecer em constante adequação a fim de se manterem competitivas ou melhorar o seu desempenho.

\subsection{Indicadores de desempenho}

Para Neely, Gregory e Platts (1996), o desempenho pode ser definido como a eficiência e a eficácia de uma ação, e a medição deste desempenho nada mais é do que métricas usadas para quantificar a eficiência e/ou eficácia da ação. No entanto, os mesmos autores destacam que medir o desempenho pode ser uma tarefa complexa, pois envolve não apenas a seleção e definição de um conjunto apropriado e prático de medidas, mas também sua integração entre si e com o ambiente em que a organização está inserida.

Rejc (2004) destacou que as organizações precisam revisar constantemente as métricas de desempenho à medida que as circunstâncias contextuais mudam, para garantir que os indicadores sejam relevantes e reflitam as questões de importância da organização. Callado, Callado, Almeida e Holanda (2012) asseguram que a escolha dos indicadores de desempenho deve estar fundamentada de maneira lógica, de modo que reflita a necessidade da empresa e que possa auxiliar os gestores de forma apropriada. 
Fatores Contingenciais e Indicadores de Desempenho: Um Estudo no Polo Calçadista de Campina Grande - PB Josélia Fernandes do Nacimento, Aldo Leonardo Cunha Callado

Sendo assim, é importante que as medidas de desempenho sejam dinâmicas para refletirem mudanças no ambiente interno e externo.

Até a década de 1990, os sistemas de mensuração de desempenho adotados pelas empresas eram compostos predominantemente por medidas financeiras. A partir de então, com a influência das modificações organizacionais, passaram a ser difundidos os indicadores que representavam medidas não financeiras, ou seja, que abordavam outras dimensões de desempenho (Diehl, Rech, \& Martins, 2016). Nesse contexto, surgiu o Balanced Scorecard (BSC) como uma metodologia de medição de desempenho que se propunha atender as necessidades das organizações modernas num processo de tomada de decisão em um ambiente ágil e que traduzisse uma visão holística da organização (Kaplan \& Norton, 1997).

$\mathrm{Na}$ visão de Kaplan e Norton (1997), as organizações modernas têm a necessidade de utilizar medidas não só financeiras, mas também medidas não financeiras para que a organização possa ter uma avaliação do alcance dos seus objetivos estratégicos de forma integrada e que contemple os objetivos de curto e longo prazo em diversas perspectivas. Ressalta-se ainda que existe uma ligação de interdependência entre as perspectivas, em que uma pode influenciar as outras. Para Kaplan e Norton (1997), os indicadores de desempenho podem ser classificados em financeiros e não financeiros e inseridos em quatro perspectivas: financeira, clientes, processos internos e aprendizagem e crescimento.

Os indicadores da perspectiva financeira indicam como as atividades estão impactando o resultado financeiro. A perspectiva com foco no cliente considera a necessidade do mesmo, pois, à medida que se percebe a importância do cliente para obter os resultados, a empresa deve buscar conseguir mantê-los no longo prazo. Com relação aos processos internos, o interesse é acompanhar os processos na busca de melhorias constantes. E, na perspectiva de aprendizagem e crescimento, a organização deve atentar para a parte física, tecnológica e intelectual da estrutura organizacional, a fim obter melhorias e maior crescimento em longo prazo (Martins, Girão, Cunha Sila, \& Araújo, 2013). É importante destacar que o BSC significa mais do que apenas utilizar 
Fatores Contingenciais e Indicadores de Desempenho: Um Estudo no Polo Calçadista de Campina Grande - PB Josélia Fernandes do Nacimento, Aldo Leonardo Cunha Callado

diversas medidas. A empresa deve considerar a importância de cada dimensão e as relações que existem entre elas.

\section{PROCEDIMENTOS METODOLÓGICOS}

A população da pesquisa compreende as empresas cadastradas no Sindicato da Indústria de Calçados do Estado da Paraíba que fazem parte do polo de Campina Grande, totalizando 30 empresas. Em virtude da indisponibilidade de dados de contato, foram excluídas 3 empresas. Sendo assim, o universo considerado para a pesquisa foi composto por 27 empresas que foram todas contactadas, sendo que 13 empresas aceitaram participar da pesquisa, correspondendo a $48,14 \%$ da população.

A pesquisa apresenta uma abordagem quantitativa, com a utilização de um questionário com base no estudo de Silva e Callado (2018). O questionário apresenta quatro blocos, divididos da seguinte forma: (i) fatores internos relacionados às características dos gestores, (ii) características relativas às empresas; (iii) fatores relacionados aos critérios de mensuração de desempenho adotados pelas organizações; e (iv) importância atribuída aos indicadores de desempenho classificados entre as dimensões do BSC. As questões sobre os fatores contingenciais foram investigadas de forma objetiva. Sobre a importância atribuída aos indicadores de desempenho, foi utilizada uma escala do tipo Likert, contendo quatro pontos para cada indicador, considerando 01 para o grau mínimo e 04 para o grau máximo de importância.

Foram considerados a faixa etária, a escolaridade e o tempo de experiência profissional como fatores contingenciais relacionados às características dos gestores. Para os fatores relacionados à empresa, consideraram-se o tempo de atuação no mercado, o número de empregados, a estrutura hierárquica e o grau de formalização dos processos operacionais. Quanto aos fatores relacionados à mensuração de desempenho, utilizou-se o critério adotado para a seleção dos indicadores de desempenho, os procedimentos referentes à mensuração e a frequência de realização 
Fatores Contingenciais e Indicadores de Desempenho: Um Estudo no Polo Calçadista de Campina Grande - PB Josélia Fernandes do Nacimento, Aldo Leonardo Cunha Callado

dos relatórios. Sobre os indicadores de desempenho investigados, classificados entre as dimensões do BSC, adotaram-se os indicadores utilizados no estudo de Silva e Callado (2018).

Para investigar as relações entre os fatores contingenciais e a importância atribuída aos indicadores de desempenho, utilizou-se o coeficiente de correlação de Spearman. As correlações que obtiveram nível de $95 \%(p=0,05)$ foram consideradas estatisticamente significantes. Foram consideradas, de acordo com Mukaka (2012), as seguintes categorias para análise de intensidade: correlação muito forte (de 0.9 a 1); correlação forte (de 0.7 a 0.9 ); correlação moderada (de 0.5 a 0.7 ); correlação fraca (de 0.3 a 0.5 ); correlação desprezível (de 0 a 0.3 ).

\section{APRESENTAÇÃO E DISCUSSÃO DOS RESULTADOS}

As primeiras relações analisadas se referem às características dos gestores. $\mathrm{Na}$ Tabela 1 são apresentados os resultados das relações dessas características na dimensão financeira.

Tabela 1

Relações entre indicadores da dimensão financeira e características dos gestores

\begin{tabular}{lccc}
\hline \multicolumn{1}{c}{ Indicadores } & Faixa Etária & Escolaridade & Experiência \\
\hline Lucratividade & $-0,358$ & 0,159 & $-0,403$ \\
Rentabilidade & $-0,459$ & $\mathbf{0 , 6 0 8 ^ { * }}$ & 0,036 \\
Crescimento das vendas & $-0,453$ & $-0,057$ & $-0,004$ \\
Custos totais & $-0,543$ & 0,083 & $-0,011$ \\
Custos unitários & 0,000 & 0,097 & 0,083 \\
Custos de entrega & $-0,305$ & $-0,060$ & $-0,128$ \\
Fluxo de caixa & $-0,232$ & 0,083 & $-0,231$ \\
Custos dos pedidos & $-0,136$ & 0,071 & 0,050 \\
Retorno do investimento & $-0,023$ & 0,350 & $\mathbf{0 , 5 6 5 ^ { \star }}$ \\
Inventários & $-0,307$ & 0,342 & $-0,070$ \\
\hline
\end{tabular}

Nota. Fonte: Dados da Pesquisa (2018).

*Nível de significância a 0,05. 
Fatores Contingenciais e Indicadores de Desempenho: Um Estudo no Polo Calçadista de Campina Grande - PB Josélia Fernandes do Nacimento, Aldo Leonardo Cunha Callado

Os resultados evidenciam que a idade do gestor não tem relação significativa com o nível de importância atribuída aos indicadores da dimensão financeira. No entanto, há correlações significativas entre a escolaridade dos gestores e o nível de importância atribuída ao indicador de rentabilidade, entre a característica de experiência profissional do gestor e o retorno de investimento. As correlações apresentam-se de forma moderada e direta. Sendo assim, os gestores com maior nível de escolaridade tendem a dar maior importância aos indicadores associados à rentabilidade do negócio, assim como os gestores com mais experiência profissional tendem a dar mais importância em questões associadas ao retorno do investimento.

De modo geral, os gestores dão relevância ao grau de êxito econômico da empresa. Segundo Tran, Nonneman e Jorissen (2014), a rentabilidade é um dos aspectos essenciais para avaliar o desempenho econômico-financeiro da organização, e os profissionais mais qualificados são fundamentais para uma boa gestão financeira.

Quando investigadas as possíveis relações entre as características dos gestores e os indicadores de desempenho da perspectiva de clientes, os resultados sugerem que há correlação significativa entre a importância atribuída à quantidade de clientes e à idade dos gestores, conforme Tabela 2 .

Tabela 2

Relações entre indicadores da dimensão clientes e características dos gestores

\begin{tabular}{lccc}
\hline \multicolumn{1}{c}{ Indicadores } & Faixa Etária & Escolaridade & Experiência \\
\hline Quantidade de clientes & $\mathbf{- 0 , 6 0 0 ^ { * }}$ & 0,000 & 0,000 \\
Novos clientes & $-0,238$ & $-0,087$ & $-0,191$ \\
Qualidade dos produtos & $-0,500$ & 0,023 & $-0,413$ \\
Preço dos produtos & $-0,282$ & $-0,336$ & 0,118 \\
Devolução de produtos & $-0,407$ & 0,264 & $-0,027$ \\
Tempo de entrega & 0,024 & 0,402 & 0,159 \\
Pontualidade de entrega & $-0,109$ & 0,496 & 0,284 \\
Responsividade & $-0,212$ & 0,427 & 0,316 \\
Crescimento da participação de & $-0,300$ & 0,255 & 0,220 \\
mercado & & & \\
\hline
\end{tabular}

Nota. Fonte: Dados da Pesquisa (2018).

*Nível de significância a 0,05. 
Fatores Contingenciais e Indicadores de Desempenho: Um Estudo no Polo Calçadista de Campina Grande - PB Josélia Fernandes do Nacimento, Aldo Leonardo Cunha Callado

Percebe-se que a correlação se apresenta inversa e moderada. Dessa forma, quando ocorre um aumento na faixa etária do gestor, há uma redução no nível de importância atribuída a questões associadas à quantidade de clientes. Para NaranjoGil, Maas e Hartmann (2009), à medida que a idade aumenta, diminui a flexibilidade e aumenta a resistência a mudanças. Cabe destacar que as características escolaridade e experiência dos gestores não apresentaram correlações com os indicadores da perspectiva de clientes.

Em seguida, foram testadas as possíveis relações entre as características dos gestores e o nível de importância dada aos indicadores de processos internos.

Tabela 3

Relações entre indicadores da dimensão processos internos e características dos gestores

\begin{tabular}{lccc}
\hline \multicolumn{1}{c}{ Indicadores } & Faixa Etária & Escolaridade & Experiência \\
\hline Tempo de realização das operações & 0,000 & $-0,221$ & $-0,234$ \\
Flexibilidade de entrega & $-0,438$ & 0,077 & 0,027 \\
Tempo de estocagem & $-0,411$ & 0,449 & 0,202 \\
Fornecedores & 0,000 & 0,431 & 0,405 \\
Flexibilidade de volume operacional & $-0,463$ & 0,021 & $-0,247$ \\
Uso da capacidade instalada & $-0,472$ & 0,390 & 0,194 \\
Pós-venda & $-0,327$ & 0,070 & $-0,04$ \\
Entregas no prazo & $-0,090$ & $-0,056$ & $-0,287$ \\
Desperdícios & $-0,327$ & $-0,221$ & $-0,061$ \\
\hline
\end{tabular}

Nota. Fonte: Dados da Pesquisa (2018).

*Nível de significância a 0,05.

De acordo com os resultados apresentados na Tabela 3, observa-se que nenhuma das características mostrou relação estatisticamente significativa, sugerindo que a importância atribuída aos indicadores dos processos internos independe da faixa etária, da escolaridade e da experiência do gestor. Este resultado também é semelhante quando investigada a possível existência de relação entre a importância dada aos indicadores de aprendizagem e crescimento e as características dos gestores, como observado na Tabela 4. 
Fatores Contingenciais e Indicadores de Desempenho: Um Estudo no Polo Calçadista de Campina

Grande - PB Josélia Fernandes do Nacimento, Aldo Leonardo Cunha Callado

Tabela 4

Relações entre indicadores da dimensão aprendizagem e crescimento e características dos gestores

\begin{tabular}{lccc}
\hline \multicolumn{1}{c}{ Indicadores } & Faixa Etária & Escolaridade & Experiência \\
\hline Produtividade dos empregados & 0,147 & $-0,219$ & 0,186 \\
Satisfação dos empregados & $-0,408$ & $-0,058$ & 0,089 \\
Rotatividade de empregados & $-0,430$ & 0,033 & 0,163 \\
Flexibilidade dos empregados & $-0,369$ & 0,132 & 0,010 \\
Inovação nos produtos & $-0,172$ & $-0,255$ & $-0,429$ \\
Investimentos em treinamento & $-0,098$ & 0,223 & $-0,190$ \\
Gestão de risco & $-0,449$ & 0,481 & 0,110 \\
\hline
\end{tabular}

Nota. Fonte: Dados da Pesquisa (2018).

*Nível de significância a 0,05.

A partir das análises acerca das relações entre características pessoais dos gestores e o nível de importância atribuída aos indicadores de desempenho, pode-se inferir que estas não são fatores preponderantes no tocante aos indicadores das dimensões de processos internos e de aprendizagem e crescimento. Hoque (2004) destaca que, por muito tempo, a alta ênfase anterior em métricas de desempenho tradicionais, como retorno sobre o investimento, desviou da preocupação devida por fatores não financeiros, como participação de mercado, satisfação do cliente, eficiência e produtividade, qualidade do produto e satisfação dos funcionários.

No estudo Chenhall e Langfield-Smith (1998), ao analisar o papel que os gestores podem desempenhar no desenvolvimento de sistemas de medição de desempenho, os autores perceberam que fatores relacionados ao nível de participação dos gestores nos processos, posição dentro da estrutura hierárquica e as habilidades técnicas e sociais podem influenciar no papel do desenvolvimento métricas de desempenho da organização. Ainda nesse sentido, Santos, Beuren, Corrêa e Martins (2018) destacam que a escolaridade e a idade do gestor são as principais características que impactam na maior utilização de ferramentas gerenciais modernas como o BSC. Segundo os mesmos autores, gestores mais jovens e gestores com maior nível de escolaridade têm maior propensão de adotar essas ferramentas. 
Fatores Contingenciais e Indicadores de Desempenho: Um Estudo no Polo Calçadista de Campina Grande - PB Josélia Fernandes do Nacimento, Aldo Leonardo Cunha Callado

O segundo bloco de fatores analisados refere-se às características das empresas. Inicialmente, foi verificada a relação entre as características das empresas e o nível de importância atribuída aos indicadores da dimensão financeira. Os resultados são apresentados na Tabela 5.

\section{Tabela 5}

Relações entre indicadores da dimensão financeira e características das empresas

\begin{tabular}{lcccc}
\hline \multicolumn{1}{c}{ Indicadores } & $\begin{array}{c}\text { Tempo de } \\
\text { Atuação }\end{array}$ & $\begin{array}{c}\text { Número de } \\
\text { Empregados }\end{array}$ & $\begin{array}{c}\text { Estrutura } \\
\text { Hierárquica }\end{array}$ & $\begin{array}{c}\text { Formalização } \\
\text { dos } \\
\text { Processos } \\
\text { Operacionais }\end{array}$ \\
\hline Lucratividade & 0,413 & 0,038 & 0,199 & 0,112 \\
Rentabilidade & 0,481 & 0,367 & $\mathbf{0 , 6 6 6 ^ { * }}$ & 0,190 \\
Crescimento das vendas & 0,200 & 0,197 & 0,518 & 0,161 \\
Custos totais & 0,220 & 0,426 & 0,541 & 0,492 \\
Custos unitários & 0,000 & $-0,213$ & 0,054 & $-0,216$ \\
Custos de entrega & 0,024 & 0,141 & 0,247 & 0,032 \\
Fluxo de caixa & 0,515 & 0,426 & 0,374 & $\mathbf{0 , 5 9 0 ^ { * }}$ \\
Custos dos pedidos & $-0,077$ & 0,032 & 0,129 & $-0,123$ \\
Retorno do investimento & 0,336 & $\mathbf{0 , 6 5 6 ^ { * }}$ & 0,508 & 0,298 \\
Inventários & 0,358 & $\mathbf{0 , 6 8 7 ^ { \star * }}$ & 0,524 & 0,165 \\
\hline
\end{tabular}

Nota. Fonte: Dados da Pesquisa (2018).

*Nível de significância a 0,05,

**Nível de significância a 0,01 .

Em relação aos resultados obtidos, constatou-se que o tempo de atuação da organização não apresenta nenhuma relação significante com a importância atribuída aos indicadores da dimensão financeira. Já o número de empregados apresenta relação estatisticamente significante a um nível moderado e de forma direta com os indicadores de retorno de investimento e inventários. Dessa forma, quanto maior o tamanho da empresa, maior será a importância correspondente a questões associadas ao retorno do investimento e a inventários.

A relação direta pode ser observada também para a associação entre estrutura hierárquica e importância atribuída à rentabilidade da empresa e a formalização dos processos, associada com o fluxo de caixa. As correlações dessas associações são consideradas moderadas. De acordo com resultados obtidos, as empresas que possuem mais níveis organizacionais e maior grau de formalização dos processos 
Fatores Contingenciais e Indicadores de Desempenho: Um Estudo no Polo Calçadista de Campina Grande - PB Josélia Fernandes do Nacimento, Aldo Leonardo Cunha Callado

indicam atribuir maior relevância aos indicadores que asseguram a continuidade da organização. Esses resultados reforçam os achados do estudo de Hoque e James (2000) que evidenciaram que quanto maior o porte da empresa, maior o uso de medidas que garantam o melhor desempenho da organização.

A Tabela 6 apresenta os resultados associados às relações entre as características das empresas e a dimensão de clientes.

Tabela 6

Relações entre indicadores da dimensão clientes e características das empresas

\begin{tabular}{|c|c|c|c|c|}
\hline Indicadores & $\begin{array}{l}\text { Tempo de } \\
\text { Atuação }\end{array}$ & $\begin{array}{l}\text { Número de } \\
\text { Empregados }\end{array}$ & $\begin{array}{l}\text { Estrutura } \\
\text { Hierárquica }\end{array}$ & $\begin{array}{c}\text { Formalização } \\
\text { dos } \\
\text { Processos } \\
\text { Operacionais }\end{array}$ \\
\hline Quantidade de clientes & 0,000 & 0,137 & 0,000 & $-0,170$ \\
\hline Novos clientes & 0,113 & $-0,037$ & 0,044 & 0,106 \\
\hline Qualidade dos produtos & 0,300 & 0,224 & 0,413 & 0,129 \\
\hline Preço dos produtos & $-0,414$ & 0,131 & 0,089 & $-0,399$ \\
\hline Devolução de produtos & 0,362 & $0,775^{\star \star}$ & $0,702^{\star *}$ & 0,423 \\
\hline Tempo de entrega & 0,231 & $0,562^{\star}$ & $0,644^{\star}$ & 0,125 \\
\hline Pontualidade de entrega & 0,260 & $0,678^{\star}$ & $0,584^{\star}$ & 0,255 \\
\hline Responsividade & 0,403 & 0,473 & $0,698^{\star \star}$ & 0,441 \\
\hline $\begin{array}{l}\text { Crescimento da participação de } \\
\text { mercado }\end{array}$ & $0,665^{\star}$ & $0,636^{\star}$ & 0,510 & $0,648^{*}$ \\
\hline
\end{tabular}

Nota. Fonte: Dados da Pesquisa (2018).

*Nível de significância a 0,05,

${ }^{* *}$ Nível de significância a 0,01.

Nesse caso, o tempo de atuação da empresa apresenta correlação positiva e significativa com o crescimento da participação do mercado e com o tamanho da empresa, representado pelo número de funcionários, sugerindo que empresas maiores e com mais experiência dão mais importância ao crescimento e participação de mercado. $O$ indicador de crescimento e participação de mercado ainda exibe associação significativa e moderada com o fator de formalização dos processos operacionais das organizações.

As variáveis número de empregados e estrutura hierárquica apresentaram correlação forte e significativa, de maneira positiva com o indicador de devolução de 
Fatores Contingenciais e Indicadores de Desempenho: Um Estudo no Polo Calçadista de Campina Grande - PB Josélia Fernandes do Nacimento, Aldo Leonardo Cunha Callado

produtos. Essas variáveis também apresentaram associação moderada com os indicadores de tempo de entrega e pontualidade de entrega de seus produtos. Além disso, a estrutura hierárquica se mostrou diretamente associada à responsividade, sugerindo que quanto mais níveis hierárquicos a empresa possuir, maior será a tendência a ter interesse em dar respostas aos clientes.

A partir desses resultados, percebe-se que as características das empresas investigadas apresentam diversas correlações estatisticamente significativas com os indicadores que se preocupam com a satisfação dos clientes. Esses resultados confrontam o estudo de Carvalho, Callado e Callado (2018), no qual o autor observou que nenhum dos indicadores de desempenho relativos ao crescimento e participação de mercado e de satisfação dos clientes apresentou associações com o tamanho e com o tempo de atuação no mercado entre as empresas investigadas.

Mediante a perspectiva de indicadores de processos internos, a análise das possíveis relações com as características das empresas está exposta na Tabela 7.

Tabela 7

Relações entre indicadores da dimensão processos internos e características das empresas

\begin{tabular}{|c|c|c|c|c|}
\hline Indicadores & $\begin{array}{l}\text { Tempo de } \\
\text { Atuação }\end{array}$ & $\begin{array}{l}\text { Número de } \\
\text { Empregados }\end{array}$ & $\begin{array}{l}\text { Estrutura } \\
\text { Hierárquica }\end{array}$ & $\begin{array}{c}\text { Formalização } \\
\text { dos } \\
\text { Processos } \\
\text { Operacionais }\end{array}$ \\
\hline $\begin{array}{l}\text { Tempo de realização das } \\
\text { operações }\end{array}$ & 0,000 & 0,531 & 0,221 & 0,378 \\
\hline Flexibilidade de entrega & 0,260 & $0,678^{\star}$ & $0,584^{\star}$ & 0,255 \\
\hline Tempo de estocagem & $0,781^{\star \star}$ & 0,424 & $0,714^{\star \star}$ & 0,383 \\
\hline Fornecedores & 0,429 & 0,213 & 0,364 & $-0,125$ \\
\hline Flexibilidade de volume operacional & 0,258 & 0,478 & $0,581^{*}$ & 0,389 \\
\hline Uso da capacidade instalada & $0,583^{\star}$ & $0,757^{\star \star}$ & $0,692^{\star \star}$ & $0,606^{*}$ \\
\hline Pós-venda & 0,155 & 0,444 & 0,447 & 0,244 \\
\hline Entregas no prazo & 0,200 & 0,506 & 0,518 & 0,412 \\
\hline Desperdícios & 0,025 & 0,547 & 0,402 & 0,524 \\
\hline
\end{tabular}

Nota. Fonte: Dados da Pesquisa (2018).

*Nível de significância a 0,05,

${ }^{* *}$ Nível de significância a 0,01. 
Fatores Contingenciais e Indicadores de Desempenho: Um Estudo no Polo Calçadista de Campina Grande - PB Josélia Fernandes do Nacimento, Aldo Leonardo Cunha Callado

Os resultados apresentados na Tabela 7 indicam correlações positivas e estatisticamente significantes entre todas as características das empresas analisadas e o nível de importância atribuído ao indicador de uso de capacidade instalada, sendo classificada como uma associação forte quanto ao número de empregados e moderada para as demais características.

Destaca-se também a associação forte na relação entre as variáveis, tempo de atuação e estrutura hierárquica com o indicador tempo de estocagem. A correlação é dada de forma direta, ou seja, quando a variável aumenta, tende a aumentar a importância ao indicador. A estrutura hierárquica ainda apresenta relações significativas e positivas com a flexibilidade de entrega e também com a flexibilidade no volume operacional. Esses resultados podem indicar que os níveis de estrutura hierárquica influenciam em questões associadas à flexibilidade das operações, além de ser o fator contingencial que mais influencia os indicadores da respectiva dos processos internos. Nesse sentido, Chenhall (2003) ressalta que à medida que a organização torna-se maior, cresce a necessidade de ter uma infraestrutura suficiente para suportar seu crescimento e de uma estrutura descentralizada capaz de lidar com uma maior quantidade de informações.

Os resultados apresentados na Tabela 8 tratam do nível de importância atribuída aos indicadores da perspectiva de aprendizagem e crescimento e a relação com as características das empresas. 
Fatores Contingenciais e Indicadores de Desempenho: Um Estudo no Polo Calçadista de Campina

Grande - PB

Josélia Fernandes do Nacimento, Aldo Leonardo Cunha Callado

Tabela 8

Relações entre indicadores da dimensão aprendizagem e crescimento e características das empresas

\begin{tabular}{lcccc}
\hline \multicolumn{1}{c}{ Indicadores } & $\begin{array}{c}\text { Tempo de } \\
\text { Atuação }\end{array}$ & $\begin{array}{c}\text { Número de } \\
\text { Empregados }\end{array}$ & $\begin{array}{c}\text { Estrutura } \\
\text { Hierárquica }\end{array}$ & $\begin{array}{c}\text { Formalização } \\
\text { dos } \\
\text { Processos } \\
\text { Operacionais }\end{array}$ \\
\hline Produtividade dos empregados & 0,280 & 0,320 & 0,255 & $\mathbf{0 , 6 4 4 ^ { \star }}$ \\
Satisfação dos empregados & 0,258 & 0,442 & 0,319 & 0,346 \\
Rotatividade de empregados & 0,204 & 0,238 & 0,303 & 0,272 \\
Flexibilidade dos empregados & 0,444 & 0,129 & 0,176 & 0,088 \\
Inovação nos produtos & 0,280 & $-0,199$ & 0,110 & $-0,049$ \\
Investimentos em treinamento & 0,444 & 0,253 & 0,431 & 0,542 \\
Gestão de risco & 0,538 & $\mathbf{0 , 6 2 8}^{\star}$ & $\mathbf{0 , 7 3 2}^{\star *}$ & 0,510 \\
\hline
\end{tabular}

Nota. Fonte: Dados da Pesquisa (2018).

*Nível de significância a 0,05,

${ }^{* *}$ Nível de significância a 0,01.

Conforme apresentado na Tabela 8, o indicador de gestão de risco apresentou correlação significativa com o número de empregados e com a estrutura hierárquica, sendo esta considerada uma forte correlação. Esses resultados podem indicar que as empresas que possuem mais empregados, ou seja, empresas maiores e que têm mais níveis hierárquicos tendem a dar maior importância à gestão de riscos. A ênfase dada ao risco das operações tornou-se uma ferramenta essencial para proteger os negócios de qualquer organização, mas especialmente as grandes empresas tendem a dedicar uma estrutura para esta atividade.

Já o indicador produtividade dos empregados apresentou significância estatística com a variável formalização dos processos operacionais. A correlação é dada de forma direta, com efeito moderado, e indica que empresas com maior grau de formalização de processos tem maior disposição de se preocupar com a produtividade dos empregados. Acredita-se que a manutenção de um alto grau de formalização das atividades resultará em níveis de produtividade cada vez mais satisfatórios. No entanto, Sefertzi (2000) afirma que a flexibilidade dos processos permite à organização se adaptar com rapidez às demandas variáveis, garantindo a competitividade do negócio. 
Fatores Contingenciais e Indicadores de Desempenho: Um Estudo no Polo Calçadista de Campina Grande - PB Josélia Fernandes do Nacimento, Aldo Leonardo Cunha Callado

Por fim, apresenta-se a análise do terceiro bloco contendo os fatores relacionados aos critérios de mensuração de desempenho adotados pelas organizações e as possíveis relações com os indicadores de desempenho e a importância atribuída. De acordo com a análise referente à dimensão financeira, os resultados indicam apenas uma correlação estatisticamente significativa. A correlação observada é forte e sugere que, à medida que há uma maior padronização dos procedimentos para mensuração de desempenho, pode aumentar a importância dada ao indicador de fluxo de caixa. Esses resultados estão dispostos na Tabela 9.

Tabela 9

Relações entre indicadores da dimensão financeira e os critérios de mensuração de desempenho adotados

\begin{tabular}{lccc}
\hline Indicadores & $\begin{array}{c}\text { Critério para } \\
\text { seleção } \\
\text { de indicadores }\end{array}$ & $\begin{array}{c}\text { Procedimentos } \\
\text { para } \\
\text { mensuração de } \\
\text { desempenho }\end{array}$ & $\begin{array}{c}\text { Frequência dos } \\
\text { relatórios sobre } \\
\text { desempenho }\end{array}$ \\
\hline Lucratividade & 0,240 & 0,262 & $-0,233$ \\
Rentabilidade & 0,228 & 0,368 & $-0,109$ \\
Crescimento das vendas & 0,323 & 0,323 & $-0,272$ \\
Custos totais & 0,276 & 0,532 & $-0,441$ \\
Custos unitários & 0,031 & 0,031 & $-0,098$ \\
Custos de entrega & 0,169 & 0,358 & $-0,547$ \\
Fluxo de caixa & 0,021 & $0,787^{\star *}$ & $-0,441$ \\
Custos dos pedidos & $-0,048$ & 0,175 & $-0,332$ \\
Retorno do investimento & $-0,189$ & 0,472 & 0,0415 \\
Inventários & 0,142 & 0,473 & $-0,134$ \\
\hline
\end{tabular}

Nota. Fonte: Dados da Pesquisa (2018).

*Nível de significância a 0,05,

**Nível de significância a 0,01.

No tocante a medidas de desempenho, o fluxo de caixa é considerado uma medida de desempenho tradicional e um dos principais instrumentos de controle gerencial (Stern, Stewart III \& Chew, 1995). Assim, o fluxo de caixa suporta a tomada de decisão quando há maior padronização e regularidade nos procedimentos referentes à mensuração do desempenho no âmbito das empresas investigadas. Os resultados apresentados demonstram que não há correlações significativas para os demais fatores. Esses resultados diferem dos resultados da pesquisa de Silva e Callado (2018), 
Fatores Contingenciais e Indicadores de Desempenho: Um Estudo no Polo Calçadista de Campina Grande - PB Josélia Fernandes do Nacimento, Aldo Leonardo Cunha Callado

na qual os critérios de mensuração de desempenho influenciaram em maior proporção no nível de importância dos indicadores da dimensão financeira do que em quaisquer outras características analisadas.

A Tabela 10 exibe as correlações dos indicadores da perspectiva clientes. Nesta perspectiva, ocorreram novamente correlações significativas apenas com o fator de formalização dos procedimentos para mensuração.

Tabela 10

Relações entre indicadores da dimensão clientes e os critérios de mensuração de desempenho adotados

\begin{tabular}{lccc}
\hline \multicolumn{1}{c}{ Indicadores } & $\begin{array}{c}\text { Critério para seleção } \\
\text { de indicadores }\end{array}$ & $\begin{array}{c}\text { Procedimentos } \\
\text { para } \\
\text { mensuração de } \\
\text { desempenho }\end{array}$ & $\begin{array}{c}\text { Frequência dos } \\
\text { relatórios sobre } \\
\text { desempenho }\end{array}$ \\
\hline Quantidade de clientes & 0,000 & 0,247 & $-0,301$ \\
Novos clientes & 0,325 & 0,286 & $-0,452$ \\
Qualidade dos produtos & 0,470 & 0,470 & $-0,458$ \\
Preço dos produtos & 0,137 & $-0,137$ & $-0,167$ \\
Devolução de produtos & 0,092 & $\mathbf{0 , 7 0 1 ^ { * * }}$ & $-0,385$ \\
Tempo de entrega & $-0,067$ & 0,395 & 0,065 \\
Pontualidade de entrega & $-0,297$ & 0,470 & $-0,004$ \\
Responsividade & $-0,175$ & 0,524 & 0,000 \\
Crescimento da participação de & 0,144 & $\mathbf{0 , 6 3 9 *}$ & $-0,075$ \\
mercado & & & \\
\hline
\end{tabular}

Nota. Fonte: Dados da Pesquisa (2018).

*Nível de significância a 0,05,

${ }^{*}$ Nível de significância a 0,01 .

As correlações estatisticamente significativas ocorrem de forma direta. Para o indicador de devolução de produtos, considera-se uma associação forte e, para o crescimento no mercado, a correlação é moderada. Diante dessas evidências, percebese que as empresas que adotam uma maior formalização para a mensuração de desempenho tendem a estar mais preocupadas com a fidelização dos clientes. Cabe destacar que a retenção de clientes atuais contribui para a maximização da participação de mercado, assim como o relacionamento com clientes e serviços de pós-venda é um 
Fatores Contingenciais e Indicadores de Desempenho: Um Estudo no Polo Calçadista de Campina Grande - PB Josélia Fernandes do Nacimento, Aldo Leonardo Cunha Callado

esforço para maximizar a satisfação do cliente, o que tende a aumentar a participação de mercado.

A seguir, a Tabela 11 apresenta os resultados de acordo com a dimensão dos processos internos.

Tabela 11

Relações entre indicadores da dimensão processos internos e os critérios de mensuração de desempenho adotados

\begin{tabular}{lccc}
\hline \multicolumn{1}{c}{ Indicadores } & $\begin{array}{c}\text { Critério para } \\
\text { seleção } \\
\text { de indicadores }\end{array}$ & $\begin{array}{c}\text { Procedimentos } \\
\text { para } \\
\text { mensuração de } \\
\text { desempenho }\end{array}$ & $\begin{array}{c}\text { Frequência dos } \\
\text { relatórios sobre } \\
\text { desempenho }\end{array}$ \\
\hline Tempo de realização das operações & 0,144 & 0,3918 & $\mathbf{- 0 , 5 7 7 ^ { * }}$ \\
Flexibilidade de entrega & 0,131 & $\mathbf{0 , 6 2 8 ^ { * }}$ & $-0,307$ \\
Tempo de estocagem & 0,260 & $\mathbf{0 , 6 1 9 ^ { * }}$ & 0,184 \\
Fornecedores & 0,031 & 0,105 & $\mathbf{0 , 7 1 9 ^ { \star * }}$ \\
Flexibilidade de volume operacional & 0,426 & $\mathbf{0 , 6 5 1}^{*}$ & $-\mathbf{0 , 6 8 3 ^ { * }}$ \\
Uso da capacidade instalada & $-0,206$ & $\mathbf{0 , 8 4 5 ^ { \star * }}$ & $-0,239$ \\
Pós-venda & 0,062 & 0,467 & $-0,264$ \\
Entregas no prazo & $\mathbf{0 , 6 2 1 ^ { * }}$ & 0,323 & $-0,2727$ \\
Desperdícios & 0,404 & 0,426 & $\mathbf{- 0 , 6 0 1 ^ { * }}$ \\
\hline
\end{tabular}

Nota. Fonte: Dados da Pesquisa (2018).

*Nível de significância a 0,05,

**Nível de significância a 0,01.

Nessa dimensão, evidenciam-se correlações fortes nas associações entre a variável, procedimentos para mensuração de desempenho e o indicador de uso da capacidade instalada, e entre a variável de frequência dos relatórios e o indicador de fornecedores. Além disso, diferentemente do que foi visto nas dimensões financeiras e de clientes, a variável frequência dos relatórios apresentou várias correlações significativas e moderadas. Ainda nessa dimensão, o critério para seleção de indicadores mostrou-se positivamente associado às entregas a prazo, indicando que, quando os indicadores são mais vinculados às metas estratégicas da empresa, possivelmente é maior o nível de importância dada às entregas no prazo.

Também foi verificado que quando há padronização e regularidade nos procedimentos de mensuração de desempenho há maior importância atribuída aos 
Fatores Contingenciais e Indicadores de Desempenho: Um Estudo no Polo Calçadista de Campina

indicadores do uso de capacidade instalada, flexibilidade no volume operacional, tempo de estocagem e flexibilidade de entrega. A flexibilidade é uma habilidade das organizações capazes de atender prontamente as mudanças e assegurar a permanência no mercado.

Por fim, analisando as possíveis relações entre os critérios de mensuração de desempenho adotados e o nível de importância conferida pelos gestores aos indicadores na perspectiva de aprendizagem e crescimento, tem-se que existe uma correlação positiva e moderada entre os critérios para a seleção de indicadores e a inovação nos produtos, podendo-se inferir que as empresas que mais dão importância à inovação dos produtos tendem a ser aquelas que possuem suas metas estratégicas vinculadas as suas escolhas.

Observou-se ainda correlação positiva e moderada quanto aos procedimentos para mensuração de desempenho com a satisfação dos empregados e a gestão de riscos. Neste caso, pode-se inferir que as organizações que buscam maior padronização e formalização na mensuração de desempenho estão mais preocupadas em adotar melhores práticas para aprimorar o desempenho da empresa a fim de que seus objetivos e metas sejam atingidos.

Os resultados associados às relações entre indicadores da dimensão aprendizagem e crescimento e os critérios de mensuração de desempenho adotados são apresentados na Tabela 12. 
Fatores Contingenciais e Indicadores de Desempenho: Um Estudo no Polo Calçadista de Campina Grande - PB Josélia Fernandes do Nacimento, Aldo Leonardo Cunha Callado

Tabela 12

Relações entre indicadores da dimensão aprendizagem e crescimento e os critérios de mensuração de desempenho adotados

\begin{tabular}{lccc}
\hline \multicolumn{1}{c}{ Indicadores } & $\begin{array}{c}\text { Critério para } \\
\text { seleção } \\
\text { de indicadores }\end{array}$ & $\begin{array}{c}\text { Procedimentos para } \\
\text { mensuração de } \\
\text { desempenho }\end{array}$ & $\begin{array}{c}\text { Frequência dos } \\
\text { relatórios sobre } \\
\text { desempenho }\end{array}$ \\
\hline Produtividade dos empregados & $-0,104$ & 0,543 & $-0,152$ \\
Satisfação dos empregados & $-0,209$ & $\mathbf{0 , 5 9 8 ^ { * }}$ & $-0,227$ \\
Rotatividade de empregados & 0,317 & 0,317 & $-0,058$ \\
Flexibilidade dos empregados & 0,099 & 0,343 & 0,282 \\
Inovação nos produtos & $\mathbf{0 , 5 6 7 ^ { * }}$ & 0,182 & 0,02 \\
Investimentos em treinamento & 0,191 & 0,414 & $-0,274$ \\
Gestão de risco & $-0,212$ & $\mathbf{0 , 6 8 3}^{\star}$ & $-0,152$ \\
\hline
\end{tabular}

Nota. Fonte: Dados da Pesquisa (2018).

*Nível de significância a 0,05,

**Nível de significância a 0,01.

Com base nos resultados obtidos para os fatores relacionados aos critérios de mensuração de desempenho, percebe-se que os procedimentos para a mensuração de desempenho configuraram o fator, dentre os analisados, que mais apresenta relações significativas, estando presente nas quatro dimensões, indicando sua relevância neste processo. Porém, diferentemente do que aponta o estudo conduzido por Silva e Callado (2018), onde os fatores relacionados aos critérios de mensuração de desempenho influenciaram em maior proporção os indicadores investigados, nesta pesquisa as características das empresas foram as que apresentaram maior número de correlações significativas, implicando assim uma maior influência nos indicadores.

Destaca-se ainda que, ao contrário ao que foi observado por Souza (2011), o presente estudo indicou que a dimensão financeira foi a que apresentou menos associações dos fatores com a importância atribuída aos indicadores.

Portanto, de acordo com o exposto, percebe-se que alguns dos fatores contingenciais considerados exercem certa associação no nível de importância atribuída pelos gestores aos indicadores de desempenho utilizados por empresas do setor calçadista de Campina Grande-PB. 
Fatores Contingenciais e Indicadores de Desempenho: Um Estudo no Polo Calçadista de Campina

Grande - PB

Josélia Fernandes do Nacimento, Aldo Leonardo Cunha Callado

\section{CONSIDERAÇÕES FINAIS}

O presente estudo buscou analisar as possíveis relações entre fatores contingenciais de indústrias do polo calçadista de Campina Grande - PB e o nível de importância atribuída pelos gestores ao uso de indicadores de desempenho, classificados mediante as perspectivas do BSC. Com base nos resultados obtidos, observou-se que as características dos gestores apresentaram poucas correlações significativas com os indicadores de desempenho, sendo estas apresentadas entre o nível de escolaridade com o indicador de rentabilidade, e uma correlação inversa entre a faixa etária dos gestores e a importância atribuída à mensuração de quantidade de clientes. Dessa forma, considera-se que as variáveis: idade, formação e experiência podem influenciar os gestores a tomarem diferentes decisões.

$\mathrm{Na}$ análise entre as características das empresas e o nível de importância atribuída aos indicadores, identificou-se a maior proporção de correlações significativas dentre todas as características estudadas, indicando associações com indicadores das quatro dimensões do BSC. Conclui-se que principalmente o tamanho e a estrutura hierárquica das organizações influenciam na utilização de práticas associadas à mensuração de desempenho.

Destaca-se ainda que, dentre os fatores investigados que discorrem sobre critérios de mensuração de desempenho, ganham destaque os procedimentos de formalização para a mensuração, estando correlacionados com o nível de importância de alguns indicadores baseados nas distintas dimensões do BSC, indicando que a padronização e regularidade dos procedimentos influenciam de maneira intensa na relevância atribuída pelos gestores aos indicadores, principalmente das perspectivas dos processos internos.

As evidências implicam ainda que os indicadores da perspectiva financeira apresentaram menos correlações com as variáveis analisadas, podendo-se inferir que as empresas investigadas possuem mais relações significativas entre os fatores investigados e o nível de importância atribuída aos indicadores não financeiros. 
Fatores Contingenciais e Indicadores de Desempenho: Um Estudo no Polo Calçadista de Campina Grande - PB Josélia Fernandes do Nacimento, Aldo Leonardo Cunha Callado

É válido enfatizar que foram tratadas características inerentes de cada empresa. Como não há uma única estrutura para as organizações, de forma análoga não há uma única forma de mensuração de desempenho, podendo cada empresa integrar seus scorecards de acordo com suas necessidades e adotando distintos indicadores. Além disso, percebe-se que não há um consenso acerca dos resultados das pesquisas por se tratarem de peculiaridades de cada organização. Ademais, o estudo também apresenta como limitação o tamanho reduzido da amostra. Assim, os resultados obtidos nesta pesquisa não podem ser generalizados e revelam apenas evidências particulares das empresas investigadas.

No entanto, esta pesquisa pode ser útil para os gestores na formulação de estratégias diante das relações analisadas. Sugere-se, portanto, que sejam realizadas investigações que tratem uma amostra maior de empresas, e ainda pode ser realizada a pesquisa de forma mais aprofundada, a fim de se buscar maior detalhamento das relações, podendo-se abranger ainda para diferentes setores ou localidades.

\section{REFERÊNCIAS}

Abicalçados. (2017). Associação Brasileira das Indústrias de Calçados - Abicalçados. Recuperado de: <http://www.abicalcados.com.br/>. Acesso em: 10/set./2018.

Beuren, I., \& Macohon, E. (2011). Institucionalização de hábitos e rotinas na contabilidade gerencial à luz da teoria da contingência: Um estudo em indústrias de móveis em São Bento do Sul. Revista de Gestão dos Países de Língua Portuguesa, 10(1-2), 78-91.

Callado, A. A. C., Callado, A. L. C., Almeida, M. A., \& de Almeida Holanda, F. M. (2012). Relacionando fatores contingenciais com o uso de indicadores de desempenho em empresas do Porto Digital. Revista de Negócios, 17(3), 20-35.

Carvalho, P. L., Callado, A. L. C., \& Callado, A. A. C. (2018). Relações entre fatores contingenciais e aspectos associados ao desempenho de empresas participantes de franquias. Contabilometria, v. 5, n. 2, p. 108-122. 
Fatores Contingenciais e Indicadores de Desempenho: Um Estudo no Polo Calçadista de Campina

Chenhall, R. H. (2003). Management control systems design within its organizational context: findings from contingency-based research and directions for the future. Accounting, organizations and society, 28(2-3), 127-168.

Chenhall, R., \& Langfield-Smith, K. (1998). Factors influencing the role of management accounting in the development of performance measures within organizational change programs. Management Accounting Research, 9(4), 361-386.

Diehl, C. A., Rech, M., \& Martins, V. Q. (2016). Indicadores Não-Financeiros de Avaliação de Desempenho: Análise de Conteúdo em Relatórios de Administração de Empresas do Setor de Distribuição de Energia Elétrica. In: Anais do XVI USP International Conference in Accounting. São Paulo, SP, Brasil, 16.

Donaldson, L., (2001). The contingency theory of organizations, Thousand Oaks, Calif., Thousand Oaks, Calif.: Sage.

Espejo, M. M. D. S. B. (2008). Perfil dos atributos do sistema orçamentário sob a perspectiva contingencial: uma abordagem multivariada. (Tese de Doutorado). Universidade de São Paulo - USP, São Paulo, SP, Brasil.

Harland, C. (1997). Supply chain operational performance roles. Integrated Manufacturing Systems, 8(2), 70-78.

Hyvönen, J. (2007). Strategy, performance measurement techniques and information technology of the firm and their links to organizational performance. Management Accounting Research, 18(3), 343-366.

Kaplan, R. S., \& Norton, D. P. (1997). A estratégia em ação: balanced scorecard. Gulf Professional Publishing.

Hoque, Z. (2004). A contingency model of the association between strategy, environmental uncertainty and performance measurement: impact on organizational performance. International Business Review, 13(4), 485-502.

Hoque, Z., \& James, W. (2000). Linking balanced scorecard measures to size and market factors: impact on organizational performance. Journal of management accounting research, 12(1), 1-17.

Martins, V. G., Girão, L. F. D. A. P., da Cunha Sila, A. C., \& Araújo, A. O. (2013). A Utilização de Modelos de Avaliação de Desempenho sob a Perspectiva do GECON e do Balanced Scorecard: Estudo de Caso em uma Central de Cooperativas de Crédito. Revista Evidenciação Contábil \& Finanças, 1(1), 99-117. 
Fatores Contingenciais e Indicadores de Desempenho: Um Estudo no Polo Calçadista de Campina

Grande - PB

Josélia Fernandes do Nacimento, Aldo Leonardo Cunha Callado

Mukaka, M. M. (2012). A guide to appropriate use of correlation coefficient in medical research. Malawi Medical Journal, 24(3), 69-71.

Naranjo-Gil, D., Maas, V. S., \& Hartmann, F. G. (2009). How CFOs determine management accounting innovation: an examination of direct and indirect effects. European accounting review, 18(4), 667-695.

Neely, A., Mills, J., Platts, K., Gregory, M., \& Richards, H. (1996). Performance measurement system design: Should process based approaches be adopted? International journal of production economics, 46, 423-431.

Otley, D. (2016). The contingency theory of management accounting and control: 19802014. Management accounting research, 31, 45-62.

Rejc, A. (2004). Toward contingency theory of performance measurement. Journal for East European Management Studies, 243-264.

Santos, V., Beuren, I. M., Corrêa, N. L., \& Martins, L. B. B. (2018). Características das empresas e dos gestores que influenciam no uso de controles gerenciais. Advances in Scientific and Applied Accounting, 11(1), 003-023.

Sefertzi, H. (2000). Os novos desenhos. HSM Management, v. 3, n. 18, p. 74-80.

Silva, M. Z., Scarpin, J. E., Rocha, W., \& Di Domenico, D. (2014). Fatores contingenciais que contribuem para a decisão de modificação do sistema de custeio: estudo de caso em uma indústria moageira. Revista de AdministraçãoRAUSP, 49(2).

Silva, A. R. D., \& Callado, A. L. C. (2018). Relações entre fatores contingenciais de empresas do setor da construção civil e a importância atribuída a indicadores de desempenho. Revista de Negócios, 17(3), 20-35.

Souza, A. E. D. (2011). Indicadores de mensuração de desempenho em pequenas e médias empresas (PMEs): estudo no setor calçadista de Santa Catarina. (Tese de Doutorado). Universidade de São Paulo - USP.

Stern, J. M., Stewart III, G. B., \& Chew, D. H. (1995). The EVA® financial management system. Journal of applied corporate finance, 8(2), 32-46.

Tran, N. M., Nonneman, W., \& Jorissen, A. (2014). Government ownership and firm performance: the case of Vietnam. International Journal of Economics and Financial Issues, 4(3), 628-650. 
Fatores Contingenciais e Indicadores de Desempenho: Um Estudo no Polo Calçadista de Campina Grande - PB Josélia Fernandes do Nacimento, Aldo Leonardo Cunha Callado

Zilber, M. A., \& Fischmann, A. A. (2002). Competitividade e a importância de indicadores de desempenho: utilização de um modelo de tendência. Encontro da Associação Nacional dos Programas de Pós-Graduação em Administração, 26.

Data de Submissão: 21/11/2019

Data de Aceite: 10/12/2019 